\title{
Outcomes of laparoscopic cholecystectomy for xanthogranulomatous cholecystitis
}

\author{
YU TAKEDA $^{1}$, YOSHITO TOMIMARU ${ }^{1}$, YUKI YOKOTA ${ }^{1}$, KOZO NOGUCHI $^{1}$, \\ SHINGO NOURA $^{1}$, HIROSHI IMAMURA ${ }^{1}$, TAKASHI IWAZAWA ${ }^{1}$, \\ KENZO AKAGI $^{1}$, SHIRO ADACHI ${ }^{2}$, TORU SHIRAKAWA ${ }^{1,3}$ and KEIZO DONO ${ }^{1}$ \\ Departments of ${ }^{1}$ Surgery and ${ }^{2}$ Pathology, Toyonaka Municipal Hospital, Toyonaka; \\ ${ }^{3}$ Department of Social Medicine, Osaka University Graduate School of Medicine, Osaka 560-8565, Japan
}

Received August 14, 2018; Accepted June 14, 2019

DOI: $10.3892 / \mathrm{mco} .2019 .1884$

\begin{abstract}
Xanthogranulomatous cholecystitis (XGC) is a rare inflammatory disease of the gallbladder with distinct histopathological characteristics. Laparoscopic cholecystectomy (LC) is currently the standard treatment for gallbladder disease. However, the outcomes of LC for XGC have not been completely investigated, due to the rarity of XGC. The present study aimed to assess the surgical outcomes of LC for XGC. Among 3,037 patients undergoing cholecystectomy between 2005 and 2017 at our institution, 58 patients (1.9\%) were diagnosed with XGC based on histopathology. Of the patients, LC was performed in $38(65.5 \%)$, and they were enrolled in the present study. The outcome of LC for XGC in the cases was assessed, and was compared with outcomes of LC for other diseases. The average operation time was $109 \pm 36 \mathrm{~min}$, and average intraoperative blood loss was $58 \pm 85 \mathrm{ml}$. LC was converted to open cholecystectomy in $6(15.8 \%)$ of the 38 cases. No operative mortality occurred. One patient developed postoperative complications greater than grade II in the Clavien-Dindo classification, and the mean postoperative hospital stay was $6.1 \pm 5.8$ days. Based on previous reports and the nature of XGC itself, the outcomes reported herein of LC for XGC seemed acceptable. It should be also noted that LC for XGC exhibited a higher conversion rate compared with $\mathrm{LC}$ than other benign gallbladder diseases, implying that $\mathrm{LC}$ for $\mathrm{XGC}$ remains challenging.
\end{abstract}

\section{Introduction}

Xanthogranulomatous cholecystitis (XGC) is an uncommon variant of chronic cholecystitis. XGC is characterized by a

Correspondence to: Dr Yoshito Tomimaru, Department of Surgery, Toyonaka Municipal Hospital, 4-14-1 Shibahara, Toyonaka, Osaka 560-8565, Japan

E-mail: ytomimaru@gmail.com

Key words: xanthogranulomatous cholecystitis, laparoscopic cholecystectomy focal or diffuse destructive inflammatory process (1-3) that frequently extends into neighboring structures. The latter characteristic results in a macroscopic appearance that is often confused with gallbladder cancer, which causes difficulties in surgical procedures. A laparoscopic cholecystectomy (LC) is currently the standard treatment for gallbladder disease. It was reported that, compared to open cholecystectomy, LC was associated with a lower postoperative complication rate and shorter postoperative hospital stay (4-6). However, few studies have investigated the surgical outcome of LC treatments for $\mathrm{XGC}$; furthermore, the outcome has been an issue of controversy (7-15). For example, in patients undergoing LC for XGC, the various reported rates of converting from laparoscopic surgery to open surgery have ranged between 10 and $80 \%$, which gave rise to the controversy. Therefore, to date, we have no unified opinion of the feasibility of LC for XGC. In addition, most previous studies were published in relatively earlier times. Considering the recent prevalence of laparoscopic surgery in fields of gastroenterologic surgery, the current conditions for performing a LC for XGC might be different from those common in previous reports. In this context, the surgical outcome of LC in patients with XGC must be newly investigated to determine the current feasibility of LC for XGC. In the present study, we aimed to assess surgical outcomes of LC for XGC, based on our experiences.

\section{Patients and methods}

A total of 3,037 patients underwent urgent cholecystectomy for gallbladder disease at the Department of Surgery, Toyonaka Municipal Hospital, between January 2005 and December 2017. Among the 3,037 patients, 58 (1.9\%) had XGC. The diagnosis of XGC was determined by experienced histopathologists, based on the following histopathological findings: the presence of diffuse or focal mural changes, with exanthema cells (foamy histiocytes containing lipids and bile pigment), giant multinucleate histiocytes, and acute or chronic inflammatory cells (1-3). These 58 patients with XGC had various preoperative diagnoses, including gallbladder stones, acute cholecystitis, chronic cholecystitis, adenomyomatosis, and gallbladder cancer (Table I). In cases with preoperative diagnosis of gallbladder cancer, we selected not laparoscopic 
approach but open approach for the cholecystectomy. In cases with preoperative diagnosis of other gallbladder diseases than gallbladder cancer, cholecystectomy was performed by laparoscopy. Exceptionally, cases with preoperative diagnosis of the other gallbladder diseases than gallbladder cancer who had concomitantly other diseases that required open abdominal surgery, open cholecystectomy was performed.

We assessed the surgical outcome of LC by comparing it to outcomes of LC for other benign gallbladder diseases. Patients with other diseases were divided into groups of urgent LC and elective LC. Urgent LC was indicated for acute cholecystitis (368 cases in the study period). Elective LC was indicated for benign gallbladder diseases, including gallbladder stones, chronic cholecystitis, and adenomyomatosis (2,377 cases in the study period).

The LC procedure was described previously (16-18). Briefly, we inserted 4 trocars. Carbon dioxide was used for peritoneal insufflation, and abdominal pressure was maintained between 8 and $12 \mathrm{mmHg}$. In cases where it is difficult to grasp the gallbladder due to its distention derived from accumulated bile or wall thickening of the gallbladder, the bile was sucked out for grasping the gallbladder. We routinely freed the cystic artery and cystic duct, achieving a critical view of safety (19), and then dissected the gallbladder. The cystic artery was sealed and dissected with laparoscopic coagulation shears, and the cystic duct was intra-abdominally ligated. In cases when the cystic duct was too thick to be ligated, vessel clip or automatic suturing device was used for closure of the cystic duct. Through the whole procedure, attention was paid to adhesion surrounding the gallbladder caused by XGC. After gallbladder dissection, the intra-abdominal cavity was washed. We defined postoperative complications as any complication that rated $\geq$ grade III in the Clavien-Dindo classification system (20).

The study protocol was approved by the Human Ethics Review Committee of Toyonaka Municipal Hospital (Certificate number: 2017-12-02). Informed consent for participation in the study was obtained through the opt-out methodology. This observational study was conducted and reported in accordance with the STROBE (Strengthening the Reporting of Observational studies in Epidemiology) guidelines $(21,22)$.

Data are expressed as the mean \pm standard deviation. Between-group differences were tested by the analysis of variance (ANOVA) for continuous variables, and by Chi-square test or Fisher's exact test for proportions. For variables showing statistical significance, post hoc tests were performed by ANOVA with Tukey's method for continuous variables and by Chi-square test or Fisher's exact test with Bonferroni's method for proportions. Statistical analyses were performed with StatView (version 5.0; SAS Institute Inc., Cary, NC, USA). P-values $<0.05$ were considered significant.

\section{Results}

Preoperative characteristics of patients with XGC. Table I summarizes the clinicopathological characteristics of all the 58 patients with XGC (34 male and 24 female; mean age $67 \pm 11$ years). The preoperative diagnoses were; gallbladder stones, $n=11$ patients; acute cholecystitis, $n=12$; chronic cholecystitis, $n=19$; adenomyomatosis, $n=2$; and gallbladder cancer, $n=14$. Among the patients with a preoperative diagnosis of chronic cholecystitis, XGC was suspected in 4. Notably, 16 patients had elevated serum CA19-9 levels $(453 \pm 1325 \mathrm{U} / \mathrm{ml})$. Among the 58 cases, 20 cases received open cholecystectomy; the open approach was selected based on preoperative suspicion of gallbladder cancer in 14 cases, and concomitant other diseases requiring open abdominal surgery in 6 cases. In the remaining 38 patients, LC was indicated. Table I also shows clinicopathological characteristics of the 20 patients with open approach and 38 patients laparoscopic approach. Due to the exclusion of patients with XGC that had a high suspicion of gallbladder cancer, the CA19-9 level in the 38 patients $(115 \pm 236 \mathrm{U} / \mathrm{ml})$ was lower than the level in the 20 patients $(981 \pm 1,985 \mathrm{U} / \mathrm{ml})$. Incidental gallbladder cancer was identified in one case among the XGC cases treated with open cholecystectomy.

Surgical outcome of LC for XGC and other diseases. Next, we compared intraoperative and postoperative factors of the 38 patients treated with LC for XGC to those of 368 patients treated with urgent $\mathrm{LC}$ and 2,377 patients treated with elective LC (Table II). The mean operative time in the 38 patients that received LC for XGC was significantly longer (109 $\pm 36 \mathrm{~min})$ than those of patients that received urgent LC $(91 \pm 33 \mathrm{~min}$; $\mathrm{P}<0.001)$ and patients that received elective LC $(72 \pm 23 \mathrm{~min}$; $\mathrm{P}<0.0001)$. There was also a significant difference in intraoperative blood loss between patients that received LC for XGC $(58 \pm 85 \mathrm{ml})$ and those that received elective LC $(12 \pm 56 \mathrm{ml}$; $\mathrm{P}=0.0003)$. Among the patients with $\mathrm{XGC}, \mathrm{LC}$ was converted to open cholecystectomy in 6 cases $(15.8 \%)$. In all 6 cases, the conversion was performed because the anatomy was obscured by severe fibrosis and bleeding around the gallbladder. The incidence of conversions in patients that received $\mathrm{LC}$ for XGC was significantly higher than that in patients that received urgent LC (4.3\%; $\mathrm{P}=0.02)$ or elective LC $(1.5 \% ; \mathrm{P}<0.0001)$. Only one patient that received LC for XGC (2.6\%) developed postoperative complications greater than grade II in the Clavien-Dindo classification. That patient exhibited an abnormal elevation of hepatobiliary enzyme, due to a common bile duct stone after LC; the stones were removed with endoscopic retrograde cholangiography. The overall incidence of complications in patients that received LC for XGC (2.6\%) was significantly higher than that of patients that received elective LC $(0.5 \% ; \mathrm{P}=0.02)$, but it was not significantly different from that of patients that received urgent $\mathrm{LC}(1.4 \% ; \mathrm{P}=0.89)$. The postoperative hospital stay among patients that received LC for XGC $(6.1 \pm 5.8$ days) was not significantly different from that of patients that received urgent LC (5.7 \pm 3.1 days), but it was significantly longer than that of patients that received elective EC (3.9 \pm 1.9 days; $\mathrm{P}<0.0001)$. No deaths occurred.

\section{Discussion}

We investigated the surgical outcome of LC in 38 patients with XGC in our institution. For reference, the outcome was compared to the outcome of LC performed in patients with other gallbladder benign diseases. Compared to both elective and urgent LC, LC for XGC was associated with a longer operation time and a higher conversion rate. In addition, compared to elective LC, LC for XGC was associated with a 
Table I. Preoperative characteristics of patients with XGC based on surgical approach.

Surgical approach for cholecystectomy

\begin{tabular}{|c|c|c|c|}
\hline \multirow[b]{2}{*}{ Factors } & \multirow[b]{2}{*}{ All XGC cases $(n=58)$} & \\
\hline & & Laparoscopic $(\mathrm{n}=38)$ & Open $(n=20)$ \\
\hline Sex (male/female) & $34 / 24$ & $24 / 14$ & $10 / 10$ \\
\hline Age (years) & $67 \pm 11$ & $67 \pm 12$ & $71 \pm 8$ \\
\hline Height (cm) & $160 \pm 9$ & $161 \pm 9$ & $158 \pm 9$ \\
\hline Weight (kg) & $61 \pm 13$ & $63 \pm 12$ & $57 \pm 14$ \\
\hline Body mass index $\left(\mathrm{kg} / \mathrm{m}^{2}\right)$ & $23.7 \pm 3.5$ & $24.2 \pm 3.0$ & $22.8 \pm 4.0$ \\
\hline ASA-PS classification $(1 / 2 / 3)$ & $12 / 35 / 11$ & $10 / 20 / 8$ & $2 / 15 / 3$ \\
\hline \multicolumn{4}{|l|}{ Laboratory data } \\
\hline $\mathrm{WBC}\left(/ \mathrm{mm}^{3}\right)$ & $7611 \pm 2824$ & $7481 \pm 2836$ & $7894 \pm 2776$ \\
\hline CRP (mg/dl) & $2.30 \pm 3.57$ & $2.13 \pm 3.69$ & $2.63 \pm 3.28$ \\
\hline Platelet $\left(10^{4} / \mathrm{mm}^{3}\right)$ & $24.6 \pm 9.9$ & $24.8 \pm 10.3$ & $24.3 \pm 9.0$ \\
\hline PT-INR & $1.11 \pm 0.17$ & $1.11 \pm 0.20$ & $1.12 \pm 0.09$ \\
\hline T-Bil (mg/dl) & $1.5 \pm 3.3$ & $0.9 \pm 0.5$ & $2.8 \pm 5.6$ \\
\hline AST (IU/1) & $41 \pm 91$ & $44 \pm 109$ & $35 \pm 27$ \\
\hline ALT (IU/l) & $37 \pm 52$ & $40 \pm 61$ & $29 \pm 20$ \\
\hline CEA (ng/ml) & $2.6 \pm 1.6$ & $2.4 \pm 1.6$ & $2.8 \pm 1.4$ \\
\hline CA19-9 (U/ml) & $453 \pm 1325$ & $115 \pm 236$ & $981 \pm 1985$ \\
\hline \multicolumn{4}{|l|}{ Preoperative diagnosis } \\
\hline Gallbladder stone & 11 & 10 & 1 \\
\hline Acute cholecystitis & 12 & 10 & 2 \\
\hline Chronic cholecystitis & 19 & 16 & 3 \\
\hline XGC suspected & 4 & 4 & 0 \\
\hline Adenomyomatosis & 2 & 2 & 0 \\
\hline Gallbladder cancer & 14 & 0 & 14 \\
\hline Gallbladder stone $(-/+)$ & $6 / 52$ & $3 / 35$ & $3 / 17$ \\
\hline Incidental gallbladder cancer $(-/+)$ & $57 / 1$ & $38 / 0$ & $19 / 1$ \\
\hline
\end{tabular}

Data are expressed as number of patients or the mean \pm standard deviation, as indicated. XGC, xanthogranulomatous cholecystitis; LC, laparoscopic cholecystectomy; ASA-PS, American Society of Anesthesiologists physical status; WBC, white blood cells; CRP, C-reactive protein; PT-INR, prothrombin time-international normalized ratio; T-Bil, total bilirubin; AST, aspartate aminotransferase; ALT, alanine aminotransferase; CEA, carcinoembryonic antigen; CA19-9, carbohydrate antigen 19-9.

higher complication rate and more intraoperative blood loss. Given the substantially different nature of XGC from that of other gallbladder benign diseases, the significant differences between LC for XGC and LC for other diseases does not deny on the feasibility of LC for XGC. Based on the concept that $\mathrm{LC}$ for the other gallbladder benign diseases is globally established surgical procedure, the comparison was intended to investigate difference between $\mathrm{LC}$ for $\mathrm{XG}$ and the established $\mathrm{LC}$ for the other gallbladder benign diseases. Thus, despite the differences in several intraoperative or postoperative factors, most of these differences were within an acceptable range, though the differences were statistically significant. The exception was the significantly higher conversion rate observed in LCs performed for XGC than in LCs performed for the other diseases. The high conversion rate in LC for XGC was due to the fact that the anatomy was obscured by severe fibrosis and bleeding around the gallbladder. This finding suggested that performing the LC for XGC was more challenging than performing the $\mathrm{LC}$ for the other diseases.
To the best of our knowledge, few studies have reported outcomes of LC for XGC (summarized in Table III) (7-15). Our study included a relatively large number of patients that underwent LC (38 cases) compared to most other studies (range, 7-47 patients). Among other studies, the range of postoperative complication rates was $0-33 \%$, with a median of 16\%; however, complications were not described in some reports. In our study, the complication rate $(2.6 \%)$ was very low compared to many previous reports. The reason for the difference in the complication rate is unclear; most of the previous studies $(7,8,10-13,15)$ did not describe definition of the complication, which might be one reason for the difference. The various conversion to open surgery rates ranged between 0 and $80 \%$ with a median of $40 \%$. Compared to those rates, ours (15.8\%) was modestly low. However, these incidences depended on each institution's criteria for LC eligibility among patients with XGC; thus, a direct comparison of incidences is not necessarily appropriate. In the present study, our criteria for LC eligibility among patients with XGC were simply that 
Table II. Surgical outcome of LC for XGC and other diseases with P-values for between-group differences.

LC for other diseases

\begin{tabular}{|c|c|c|c|c|}
\hline \multirow[b]{2}{*}{ Outcome measures } & \multirow[b]{2}{*}{ LC for $\mathrm{XGC}(\mathrm{n}=38)$} & & \multirow[b]{2}{*}{ P-value } \\
\hline & & Urgent LC $(n=368)$ & Elective LC (n=2377) & \\
\hline \multicolumn{5}{|l|}{ Preoperative factors } \\
\hline Sex (male/female) & $24 / 14$ & $237 / 131$ & $1086 / 1291^{\mathrm{a}}$ & $<0.0001$ \\
\hline Age (years) & $65 \pm 13$ & $64 \pm 15$ & $60 \pm 14$ & $<0.0001$ \\
\hline \multicolumn{5}{|l|}{ Intraoperative factors } \\
\hline Operation time (min) & $109 \pm 36$ & $91 \pm 33^{c}$ & $72 \pm 23^{c}$ & $<0.0001$ \\
\hline Intraoperative blood loss (ml) & $58 \pm 85$ & $51 \pm 133$ & $12 \pm 56^{c}$ & $<0.0001$ \\
\hline Conversion to open surgery $(-/+)$ & $32 / 6$ & $352 / 16^{\mathrm{b}}$ & $2341 / 36^{c}$ & $<0.0001$ \\
\hline \multicolumn{5}{|l|}{ Postoperative factors } \\
\hline Mortality (-/+) & $38 / 0$ & $368 / 0$ & $2377 / 0$ & $>0.9999$ \\
\hline Complication $(-/+)^{\mathrm{d}}$ & $37 / 1$ & $363 / 5$ & $2364 / 13^{c}$ & 0.03 \\
\hline Bleeding & 0 & 0 & 1 & \\
\hline Cerebral infarction & 0 & 0 & 1 & \\
\hline Respiratory diseases & 0 & 1 & 1 & \\
\hline Bile leakage & 0 & 0 & 3 & \\
\hline Intra-abdominal abscess & 0 & 0 & 3 & \\
\hline CBD stone or cholangitis & 1 & 2 & 2 & \\
\hline Ileus & 0 & 1 & 0 & \\
\hline Other & 0 & 1 & 2 & \\
\hline Postoperative hospital stay (days) & $6.1 \pm 5.8$ & $5.7 \pm 3.1$ & $3.9 \pm 1.9^{c}$ & $<0.0001$ \\
\hline
\end{tabular}

Table III. Data from previous studies on LC for XGC.

\begin{tabular}{|c|c|c|c|c|c|c|c|c|}
\hline Study & Authors & Year & $\begin{array}{l}\text { Number of } \\
\text { XGC cases }\end{array}$ & $\begin{array}{c}\text { Number of } \\
\text { LC cases }(\%)\end{array}$ & Conversion (\%) & Mortality (\%) & Morbidity (\%) & (Refs.) \\
\hline 1 & Guzman-Valdivia & 2004 & 182 & $4 \quad(23)$ & $33(80)$ & $0(0)$ & $9(22)$ & (12) \\
\hline 2 & Srinivas, et al & 2007 & 29 & $24 \quad(83)$ & $11(46)$ & $0(0)$ & $6(21)^{a}$ & (10) \\
\hline 3 & Kim, et al & 2009 & 23 & $15 \quad(65)$ & $6(40)$ & $0(0)$ & $2(13)$ & (15) \\
\hline 4 & Wang, et al & 2009 & 74 & $47 \quad(64)$ & $5(11)$ & $0(0)$ & $0(0)$ & (11) \\
\hline 5 & Jetley, et al & 2012 & 13 & $13(100)$ & $8(62)$ & N/A & N/A & (14) \\
\hline 6 & Han and Chen & 2012 & 39 & $7 \quad(18)$ & $2(29)$ & $0(0)$ & $4(10)^{\mathrm{a}}$ & (13) \\
\hline 7 & Alvi, et al & 2013 & 27 & $17 \quad(63)$ & $9(53)$ & $0(0)$ & $0(0)$ & (7) \\
\hline 8 & Qasaimeh, et al & 2015 & 42 & $35 \quad(83)$ & $11(31)$ & $0(0)$ & $14(33)^{\mathrm{a}}$ & (8) \\
\hline 9 & $\begin{array}{l}\text { Park JW, et al } \\
\text { The present study }\end{array}$ & 2017 & $\begin{array}{l}31 \\
58\end{array}$ & $\begin{array}{l}31(100) \\
38(66)\end{array}$ & $\begin{array}{l}5(16) \\
6(16)\end{array}$ & $\begin{array}{l}0(0) \\
0(0)\end{array}$ & $\begin{array}{l}6(19) \\
1(3)\end{array}$ & (9) \\
\hline
\end{tabular}

${ }^{a}$ The numbers and percentages of XGC cases refer to all XGC cases, including patients with XGC that underwent open cholecystectomy. XGC, xanthogranulomatous cholecystitis; LC, laparoscopic cholecystectomy; N/A, not assigned.

patients should have no high suspicion of gallbladder cancer and no other diseases that required open abdominal surgery. Thus, our criteria for LC were not overly restrictive. In this context, our findings for the outcomes of LC for XGC might be considered better than the findings in previous reports.
There have been several clinical problems of XGC when practically dealing with $\mathrm{XGC}$; one is, as commented above, difficulty of surgical treatment due to adhesion surrounding the gallbladder caused by XGC. Another problem is difficulty of differential diagnosis of $\mathrm{XGC}$ from gallbladder cancer 
based on preoperative imagings. In the present study, sensitivity of preoperative imagings for the XGC diagnosis was only $6.9 \%$ (4 cases out of the 58 cases), though sensitivity of preoperative imagings for the diagnosis of gallbladder cancer was not investigated in this study since this study did not include patients who were diagnosed with gallbladder cancer both preoperatively and histopathologically. In addition, $24.1 \%$ (14 cases out of the 58 cases) of the XGC cases had been diagnosed with gallbladder cancer. These results also problematize the difficulty of differential diagnosis. The other is high frequency of coincidence of gallbladder cancer with XGC. We also experienced one case (1.7\%) with the coexistence, while the frequency seemed to be lower than previous studies $(2,7,23,24)$. At the surgery for XGC, we should keep possibility of the coexistence in mind.

The present study have several limitations. First, although our sample size was actually larger than those in most previous reports, it was relatively small, and all patients were enrolled at a single institution. Studying data from a single institution might have introduced some bias in the surgical outcome findings, due to the abovementioned discrepancy among different institutions in the criteria for LC eligibility for patients with XGC. This limitation could be overcome by recruiting more patients with XGC from multiple institutions. Also, associated with the abovementioned LC eligibility criteria, our study focused only on patients that underwent surgery with LC; thus, patients with XGC that did not require surgery were not included in the analysis. Another limitation was the 'retrospective' design of the study. All data were collected from information obtained from medical records. Therefore, the results should be validated in a larger prospective study. Another limitation that applies to all studies on patients diagnosed with XGC is the ambiguity of histopathological diagnostic criteria for $\mathrm{XGC}$. The universal criteria for XGC are the presence of diffuse or focal mural changes with the presence of so-called exanthema cells, giant multinucleate histiocytes, and inflammatory cells. However, there are no strict criteria for the extent of the pathologic cells in the gallbladder. For example, in some cases, the histopathology is detected throughout the entire gallbladder; these cases are unambiguously diagnosed as XGC. However, it is not clear whether a XGC diagnosis is warranted when only a very small area of the gallbladder exhibits the histopathology of XGC. This ambiguity in diagnostics could affect the incidence of XGC and contribute to the difficulty of XGC surgery. To address this problem, clear diagnostic criteria for XGC should be firmly established.

In the present study, we assessed surgical outcomes of LC for XGC and compared the outcomes with those of patients with LC for other gallbladder benign diseases based on our experiences. As a result, LC for XGC exhibited higher conversion rate compared to $\mathrm{LC}$ for other gallbladder benign diseases. On the other hands, the higher conversion rate in our case series was modestly lower than previous studies Furthermore, the complication rate in our case series was very low compared to the previous reports. These results suggested that $\mathrm{LC}$ for $\mathrm{XGC}$ is both challenging and relatively acceptable.

\section{Acknowledgements}

Not applicable.

\section{Funding}

No funding was received.

\section{Availability of data and materials}

All data and materials in this study are included in this published article.

\section{Authors' contributions}

YTa and YTo were responsible for the concept and design of the study and for data interpretation. YY, SN, HI, TI and KN assisted in data interpretation. SN, HI, TI, and KA assisted in articles preparation. SA supported histological diagnosis. TS assisted in statistics. KD supervised the whole project. All authors read and approved the final manuscript.

\section{Ethics approval and consent to participate}

Ethical approval for this study was obtained from the Human Ethics Review Committee of Toyonaka Municipal Hospital (Certificate Number 2017-12-02).

\section{Patient consent for publication}

We confirm that informed consent for participation was obtained.

\section{Competing interests}

The authors declare that they have no competing interests.

\section{References}

1. Houston JP, Collins MC, Cameron I, Reed MW, Parsons MA and Roberts KM: Xanthogranulomatous cholecystitis. Br J Surg 81: 1030-1032, 1994

2. Goodman ZD and Ishak KG: Xanthogranulomatous cholecystitis Am J Surg Pathol 5: 653-659, 1981.

3. Christensen AH and Ishak KG: Benign tumors and pseudotumors of the gallbladder. Report of 180 cases. Arch Pathol 90: 423-432, 1970.

4. Kiviluoto T, Siren J,Luukkonen P and Kivilaakso E: Randomised trial of laparoscopic versus open cholecystectomy for acute and gangrenous cholecystitis. Lancet 351: 321-325, 1998.

5. Coccolini F, Catena F, Pisano M, Gheza F, Fagiuoli S, Di Saverio S, Leandro G, Montori G, Ceresoli M, Corbella D, et al: Open versus laparoscopic cholecystectomy in acute cholecystitis. Systematic review and meta-analysis. Int J Surg 18: 196-204, 2015.

6. Boo YJ, Kim WB, Kim J, Song TJ, Choi SY, Kim YC and Suh SO: Systemic immune response after open versus laparoscopic cholecystectomy in acute cholecystitis: A prospective randomized study. Scand J Clin Lab Invest 67: 207-214, 2007.

7. Alvi AR, Jalbani I, Murtaza G and Hameed A: Outcomes of Xanthogranulomatous cholecystitis in laparoscopic era: A retrospective Cohort study. J Minim Access Surg 9: 109-115, 2013.

8. Qasaimeh GR, Matalqah I, Bakkar S, Al Omari A and Qasaimeh M: Xanthogranulomatous cholecystitis in the laparoscopic era is still a challenging disease. J Gastrointest Surg 19: 1036-1042,2015.

9. Park JW, Kim KH, Kim SJ and Lee SK: Xanthogranulomatous cholecystitis: Is an initial laparoscopic approach feasible? Surg Endosc 31: 5289-5294, 2017.

10. Srinivas GN, Sinha S, Ryley N and Houghton PW: Perfidious gallbladders - a diagnostic dilemma with xanthogranulomatous cholecystitis. Ann R Coll Surg Engl 89: 168-172, 2007. 
11. Wang M, Zhang T, Zang L, Lu A, Mao Z, Li J, Dong F, Hu W, Jiang $\mathrm{Y}$ and Zheng M: Surgical treatment for xanthogranulomatous cholecystitis: A report of 74 cases. Surg Laparosc Endosc Percutan Tech 19: 231-233, 2009.

12. Guzman-Valdivia G: Xanthogranulomatous cholecystitis in laparoscopic surgery. J Gastrointest Surg 9: 494-497, 2005.

13. Han SH and Chen YL: Diagnosis and treatment of xanthogranulomatous cholecystitis: A report of 39 cases. Cell Biochem Biophys 64: 131-135, 2012.

14. Jetley S,RanaS,KhanRNand JairajpuriZS:Xanthogranulomatous cholecystitis-a diagnostic challenge. J Indian Med Assoc 110 833-837, 2012.

15. Kim JH, Jeong IH, Yoo BM, Kim JH, Kim MW and Kim WH: Is xanthogranulomatous cholecystitis the most difficult for laparoscopic cholecystectomy? Hepatogastroenterology 56: 597-601, 2009.

16. Noda T, Hatano H, Dono K, Shimizu J, Oshima K, Tanida T, Miyake M, Komori T, Kawanishi K, Morita S, et al: Safety of early laparoscopic cholecystectomy for patients with acute cholecystitis undergoing antiplatelet or anticoagulation therapy: A single-institution experience. Hepatogastroenterology 61: 1501-1506, 2014.

17. Yoshihara T, Tomimaru Y, Noguchi K, Nagase H, Hamabe A, Hirota M, Oshima K, Tanida T, Morita S, Imamura H, et al: Feasibility of laparoscopic cholecystectomy in patients with cerebrospinal fluid shunt. Asian J Endosc Surg 10: 394-398, 2017.

18. Shinke G, Noda T, Hatano H, Shimizu J, Hirota M, Takata A Oshima K, Tanida T, Komori T, Morita S, et al: Feasibility and safety of urgent laparoscopic cholecystectomy for acute cholecystitis after 4 days from symptom onset. J Gastrointest Surg 19: $1787-1793,2015$
19. Strasberg SM, Hertl M and Soper NJ: An analysis of the problem of biliary injury during laparoscopic cholecystectomy. J Am Coll Surg 180: 101-125, 1995

20. Dindo D, Demartines N and Clavien PA: Classification of surgical complications: A new proposal with evaluation in a cohort of 6,336 patients and results of a survey. Ann Surg 240: 205-213, 2004.

21. Vandenbroucke JP, von Elm E, Altman DG, Gøtzsche PC, Mulrow CD, Pocock SJ, Poole C, Schlesselman JJ and Egger M; STROBE Initiative: Strengthening the reporting of observational studies in epidemiology (STROBE): Explanation and elaboration. PLoS Med 4: e297, 2007.

22. von Elm E, Altman DG, Egger M, Pocock SJ, Gotzsche PC and Vandenbroucke JP; STROBE Initiative: The strengthening the reporting of observational studies in epidemiology (STROBE) statement: Guidelines for reporting observational studies. Lancet 370: 1453-1457, 2007.

23. Ghosh M, Sakhuja P and Agarwal AK: Xanthogranulomatous cholecystitis: A premalignant condition? Hepatobiliary Pancreat Dis Int 10: 179-184, 2011.

24. Yang T, Zhang BH, Zhang J, Zhang YJ, Jiang XQ and Wu MC: Surgical treatment of xanthogranulomatous cholecystitis: Experience in 33 cases. Hepatobiliary Pancreat Dis Int 6: 504-508, 2007. 